5. Zavadil, J., and Bottinger, E.P. 2005. TGF-beta and epithelial-to-mesenchymal transitions. Oncogene. 24:5764-5774.

6. Iwano, M., et al. 2002. Evidence that fibroblasts derive from epithelium during tissue fibrosis. J. Clin. Invest. 110:341-350.

7. Nawshad, A., Lagamba, D., Polad, A., and Hay, E.D. 2005. Transforming growth factor-beta signaling during epithelial-mesenchymal transformation: implications for embryogenesis and tumor metastasis. Cells Tissues Organs. 179:11-23.

8. Radisky, D.C., et al. 2005. Rac1b and reactive oxygen species mediate MMP-3-induced EMT and genomic instability. Nature. 436:123-127.

9. Nelson, W.J., and Nusse, R. 2004. Convergence of Wnt, beta-catenin, and cadherin pathways. Science. 303:1483-1487.

10. Cadigan, K.M. 2008. Wnt-beta-catenin signaling. Curr. Biol. 18:R943-R947.

11. Lilien, J., and Balsamo, J. 2005. The regulation of cadherin-mediated adhesion by tyrosine phosphorylation/dephosphorylation of beta-catenin. Curr. Opin. Cell Biol. 17:459-465.

12. Masszi, A., et al. 2004. Integrity of cell-cell contacts is a critical regulator of TGF-beta 1-induced epithelial-to-myofibroblast transition: role for betacatenin. Am. J. Pathol. 165:1955-1967.

13. Huber, A.H., Stewart, D.B., Laurents, D.V., Nelson, W.J., and Weis, W.I. 2001. The cadherin cytoplasmic domain is unstructured in the absence of beta-catenin. A possible mechanism for regulating cadherin turnover. J. Biol. Chem. 276:12301-12309.

14. Roura, S., Miravet, S., Piedra, J., Garcia de Herreros, A., and Dunach, M. 1999. Regulation of E-cadherin/ Catenin association by tyrosine phosphorylation. J. Biol. Chem. 274:36734-36740.

15. Kim, K., Lu, Z., and Hay, E.D. 2002. Direct evidence for a role of beta-catenin/LEF-1 signaling pathway in induction of EMT. Cell. Biol. Int. 26:463-476.

16. Selman, M., Pardo, A., and Kaminski, N. 2008. Idio- pathic pulmonary fibrosis: aberrant recapitulation of developmental programs? PLoS Med. 5:e62.

17. Konigshoff, M., et al. 2008. Functional Wnt signaling is increased in idiopathic pulmonary fibrosis. PLOS ONE. 3:e2142.

18. Medici, D., Hay, E.D., and Goodenough, D.A. 2006. Cooperation between snail and LEF-1 transcription factors is essential for TGF-beta1-induced epithelial-mesenchymal transition. Mol. Biol. Cell. 17:1871-1879.

19. Chattopadhyay, N., Wang, Z., Ashman, L.K., Brady-Kalnay, S.M., and Kreidberg, J.A. 2003. alpha3beta1 integrin-CD151, a component of the cadherin-catenin complex, regulates PTPmu expression and cell-cell adhesion. J. Cell Biol. 163:1351-1362.

20. Kim, K.K., et al. 2009. Epithelial cell $\alpha 3 \beta 1$ integrin links $\beta$-catenin and Smad signaling to promote myofibroblast formation and pulmonary fibrosis. J. Clin. Invest. 119:213-224.

\title{
Revisiting Notch in remyelination of multiple sclerosis lesions
}

\author{
Celia F. Brosnan ${ }^{1}$ and Gareth R. John²
}

\begin{abstract}
${ }^{1}$ Department of Pathology and Department of Neuroscience, Albert Einstein College of Medicine, New York, New York, USA. ${ }^{2}$ Corinne Goldsmith Dickinson Center for Multiple Sclerosis, Department of Neurology, Mount Sinai School of Medicine, New York, New York, USA.
\end{abstract}

\begin{abstract}
MS results from destruction of the protective myelin sheath surrounding axons, which prevents the transmission of nerve impulses. Precursors of oligodendrocytes, the cells capable of myelinating axons, are preserved in demyelinating lesions; however, why these precursors do not differentiate into mature oligodendrocytes and remyelinate axons is unknown. Contactin is a noncanonical Notch receptor ligand that mediates oligodendrocyte differentiation. In this issue of the JCI, Nakahara et al. show that Contactin is abundantly expressed on demyelinated axons in human chronic MS lesions and that Notch1 is activated in oligodendrocyte precursor cells (see the related article beginning on page 169). However, Notch1 intracellular domain coassociates with the nuclear transporter Importin $\beta$ but fails to show evidence of nuclear translocation. These cytoplasmic aggregates also contain TAT-interacting protein $30 \mathrm{kDa}$ (TIP30), a proapoptotic factor, which inhibits nuclear transport and, consequently, Notch1-mediated oligodendrocyte differentiation and remyelination. These data target TIP30 as a new pathogenic factor in MS.
\end{abstract}

MS is an inflammatory, demyelinating disease of the CNS that is thought to be mediated by an immune attack directed against oligodendrocytes and myelin. Clinically, patients usually present in early adulthood with a relapsing/remitting form of the disease that over time develops into a chronic

Conflict of interest: The authors have declared that no conflict of interest exists.

Nonstandard abbreviations used: DSL, Delta, Serrate/Jagged, Lag2; HES1, hairy and enhancer of split 1 ; NICD, Notch intracellular domain; OPC, oligodendrocyte precursor cell; PLP, myelin proteolipid protein; TIP30, TAT-interacting protein $30 \mathrm{kDa}$.

Citation for this article: J. Clin. Invest. 119:10-13 (2009). doi:10.1172/JCI37786. progressive state with increasing disability. Pathological staging of MS lesions remains controversial, but they are usually categorized as active/acute, chronic active, and chronic inactive/silent. Acute and chronic active lesions are characterized by perivascular cuffs of lymphocytes and macrophages associated with areas of primary demyelination, with chronic active lesions often showing a chronic core and an active edge, whereas chronic silent lesions display loss of oligodendrocytes, myelin, and axons, with little to no evidence of ongoing inflammation (1). At the edge of some lesions, substantial numbers of premyelinating oligodendrocytes can be observed, indicating that the potential for repair is not limited by the loss of these cells but rather reflects gain or loss of signals that promote oligodendrocyte migration into lesioned areas of the brain and their differentiation into mature myelinating oligodendrocytes. Remarkably, all three types of lesions may be found within adjacent areas of the same brain, suggesting that it is the microenvironment of the lesion itself that influences lesion outcome.

Notch receptor family members (Notch1Notch4) are type 1 transmembrane proteins that, following ligand binding, are cleaved by a metalloproteinase and a $\gamma$-secretase, generating Notch intracellular domain (NICD), which then translocates to the nucleus where it acts as a second messenger to modulate expression of target genes (2). Depending upon the ligand involved in Notch1 activation, these target genes may either inhibit (via canonical signaling) or promote (via noncanonical signaling) differentiation and maturation of oligodendrocyte precursor cells (OPCs) (Figure 1). Ligands belonging to the canonical pathway, such as Delta, Serate/Jagged, and Lag2 (DSL), transduce signals through the CSL/ NICD/Mastermind (where CSL represents $\mathrm{CBF} 1, \mathrm{Su}[\mathrm{H}], \mathrm{Lag} 1)$ signaling pathway, leading to transcriptional activation of the inhibitory genes hairy and enhancer of split 1 (HES1), HES5, and HES7 as well as hairy and enhancer of split related with YRPW 


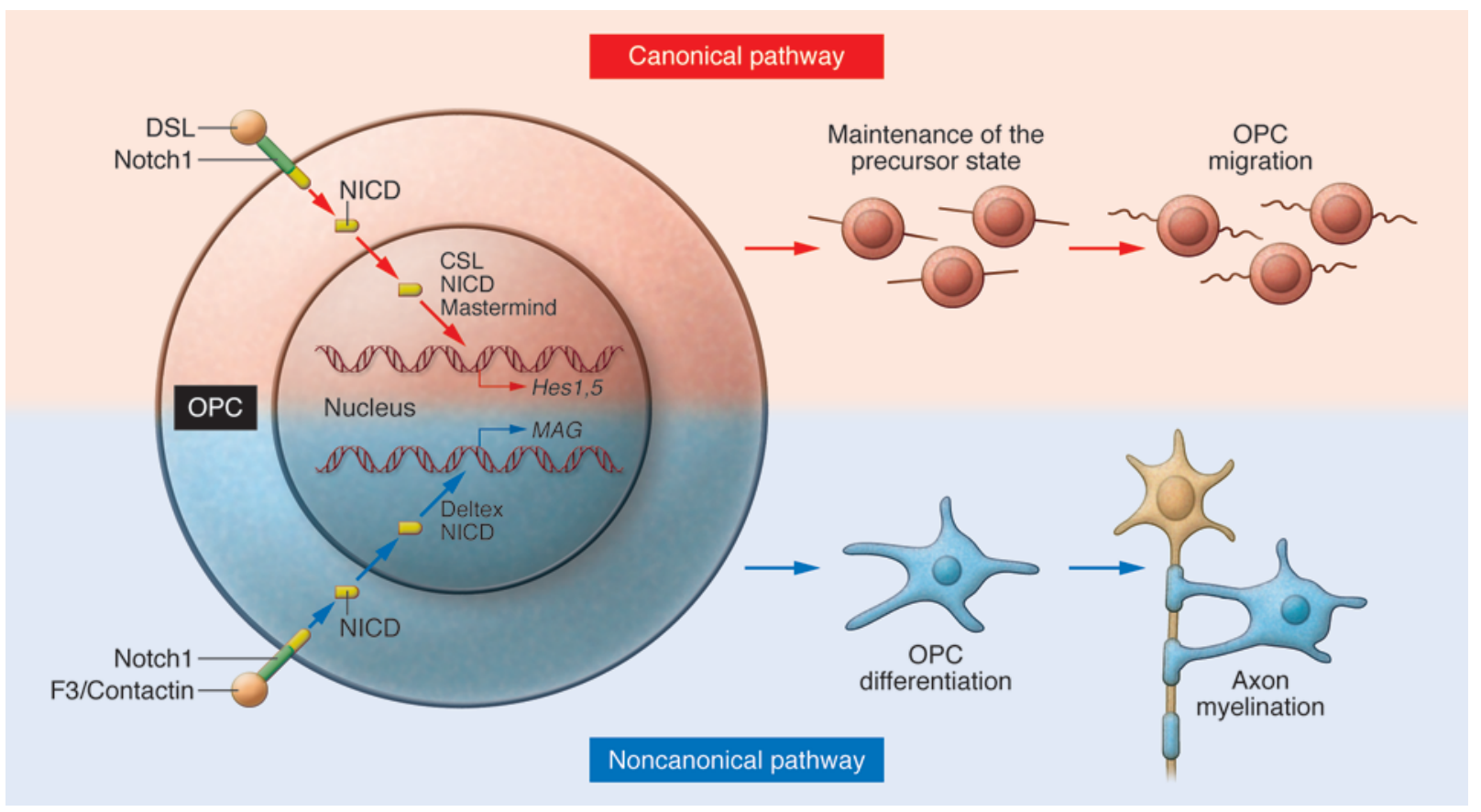

\section{Figure 1}

Notch1 ligands differentially regulate OPC development. The figure depicts the outcome of the interaction of different ligands with the Notch1 receptor, which triggers signaling via two distinct pathways. Canonical ligands of the DSL family activate genes such as HES1 and HES5 $(H E S 1,5)$ that inhibit OPC differentiation but permit OPC proliferation, resulting in the maintenance and/or expansion of the OPC pool. In contrast, the noncanonical ligand F3/Contactin leads to OPC differentiation and subsequent myelination of axons by promoting expression of myelin genes such as myelin-associated glycoprotein (MAG). However, in this issue of the $\mathrm{JCl}$, Nakahara et al. (8) present data supporting the conclusion that in MS lesions, activation of the noncanonical pathway may fail due to pathologic upregulation of TIP30, a protein that blocks nuclear transport of NICD. NICD entrapment in the cytoplasm prevents OPC differentiation and axon myelination (see schematic representation depicted in Figure 9 of the Nakahara et al. study; ref. 8).

motif 1 (HEY1), HEY2, and HEYL, which block OPC maturation, resulting in maintenance of the OPC pool (2-4). In contrast, ligands of the noncanonical pathway, such as F3/Contactin, transduce signals through the NICD/Deltex signaling cascade, leading to transcriptional activation of genes such as myelin-associated glycoprotein $(M A G)$ promoting OPC differentiation and myelination (Figure 1) $(2,5)$. During development, expression of Notch1 and ligands of both the canonical and noncanonical pathways are temporally and spatially controlled to coordinate commitment of cells to the oligodendrocyte lineage, proliferation and maintenance of OPCs, and differentiation of OPCs into mature myelinating oligodendrocytes $(2,6)$.

\section{Notch signaling in chronic MS lesions}

In earlier studies, we examined chronic active MS lesions for expression of members of the Notch canonical signaling pathway and found expression of Jagged 1 by reactive hypertrophic astrocytes and
Notch1 and nuclear HES5 in OPCs (7). Now, in this issue of the JCI, Nakahara and colleagues have examined human chronic silent MS lesions for components of the noncanonical Notch signaling pathway (8). They hypothesized that, since axons are a major site of F3/Contactin expression and since axonal damage is a feature of chronic inactive MS lesions, remyelination failure in these lesions might reflect loss of a Contactin/Notch1-dependent activation of oligodendrocyte differentiation. Surprisingly, however, they found that Contactin was abundantly expressed in chronic, demyelinated MS lesions, with enhanced immunohistochemical staining for Contactin clearly demarcating affected regions of the brain. Furthermore, they detected NICD within the cytoplasm of OPCs, thus providing evidence of Notch 1 activation at these sites. Detection of cytoplasmic NICD is unusual since, once activated, NICD rapidly translocates to the nucleus. To follow up on this unexpected finding, Nakahara and colleagues tested for expression of factors involved in the nuclear translocation process. These studies revealed that NICD colocalized with the nuclear transporter Importin $\beta$ and the nuclear pore protein Nup153. What is perhaps even more interesting is that these aggregates also coassociated with Lamin B1, which was also overexpressed in the demyelinated MS lesions. Duplications and overexpression of Lamin B1 have recently been identified as the cause of autosomal dominant leukodystrophy, a slowly progressive adult-onset demyelinating disease (9), which has often been misdiagnosed as chronic, progressive MS but which differs pathologically in that oligodendrocytes are preserved and there is no astrogliosis (10). Seeking a mechanism whereby these proteins may get trapped in the cytoplasm, Nakahara et al. then examined expression of TATinteracting protein $30 \mathrm{kDa}$ (TIP30, also known as CC3), a protein known to form a complex with Importin $\beta$ and to block its nuclear translocation (11). Again, they found increased expression of TIP30 in demyelinated lesions and showed association with aggregates of Importin B1. 
This study by Nakahara et al. (8) is important in that it is the first to examine components of a noncanonical Notch1 signaling pathway in MS lesions. The results clearly show that OPCs are present in chronic lesions, that they express Notch 1 , and that Notch 1 signaling is activated. The authors did not detect a strong signal for nuclear HES5, suggesting that the canonical pathway was not activated in these lesions, in contrast to our previous report (7). A likely explanation for these differences is that whereas Nakahara et al. examined chronic silent MS lesions, the plaques in our study were predominantly chronic active. That chronic active and chronic silent MS lesions may differentially display transcribed genes has been well documented by microarray analysis (12). These differences might, therefore, reflect a switch from an environment where expansion of the OPC pool is encouraged via signaling through the canonical pathway to one that directs their differentiation and entry into the myelination program via signaling through the noncanonical pathway. Taken together, these studies suggest that all the machinery for remyelination is present in the MS lesion but that myelination in some lesions fails due to a block in signaling mediated by pathologic expression of TIP30. Nakahara and colleagues further support this conclusion by showing reduced expression of NICD/TIP30 cytoplasmic aggregates in remyelinated shadow plaques as well as studies in vitro using the OPC cell line OLN39, in which transfection with an enhanced GFP-tagged TIP30 vector blocked Contactin-induced differentiation. Furthermore, in line with the known proapoptotic properties of TIP30, many of the TIP30transfected cells died (8). From these data, the authors conclude that OPCs in chronic MS lesions are not naive but pathogenic.

\section{Potential role of TIP30 in MS pathogenesis}

TIP30 is a normal cellular protein originally identified as a tumor suppressor that, when present in excess, blocks nuclear import $(11,13)$. It is known to be upregulated by heat shock, stress, aging, certain viruses, and the cytokine TGF- $\beta$. The tumor-suppressive activity of TIP30 has been linked to the induction of apoptosis, possibly by blocking of nuclear pores (11), and the suppression of osteopontin (14), a proinflammatory cytokine that has been implicated in the generation of Th17 cells, important for the generation of cell-mediated immunity. Osteopontin has been shown to be upregulated in chronic active MS lesions and has been linked to the exacerbation of the animal model for MS known as EAE (15). Thus, these studies reported by Nakahara et al. (8) introduce a new player into the pathogenesis of MS, and it will be of considerable interest to determine whether TIP30 plays a role in the pathogenesis of animal models of demyelination/remyelination and, in particular, of EAE.

\section{Contactin expression in the CNS}

These studies by Nakahara et al. (8) are also important in that they show that a member of the noncanonical pathway, Contactin, is reexpressed in the injured adult CNS. OPC expansion in response to canonical Notch1 ligands such as Jagged1 is potentially beneficial in MS, assuming that the cells in the resulting pool remain sensitive to the signals that then drive their differentiation and myelin formation. During development, DSL ligands are expressed early, whereas Contactins are highly expressed only after birth. Contactins are glycosylphosphatidylinisotol-linked (GPIlinked) members of the immunoglobulin superfamily (16). Neurons express two isoforms that differ in the extent of glycosylation, with the lower MW isoform colocalizing with Contactin-associated protein (Caspr) at paranodes and the higher MW isoform detected in the nodal region (17). In contrast with Contactin, Caspr has been reported to be lost in chronic demyelinated MS lesions (18). Thus, another critical question that will require further investigation is how Contactin expression is regulated on demyelinated axons.

\section{Future directions}

Studies of appropriate animal models and more detailed analyses of MS lesions that do successfully remyelinate may help to address these issues. With respect to animal models, EAE is not ideal for deciphering the role of Notch 1 in remyelination since Notch signaling also regulates cells of the immune system (19). Nonimmune demyelinating/remyelinating animal models involve stereotactic microinjection of lysolecithin or ethidium bromide or chronic ingestion of the copper chelator cuprizone. Cuprizone leads to selective demyelination/remyelination of the corpus callosum and cerebellar peduncles, whereas microinjection models are usually targeted either to the dorsal columns of the spinal cord or the corpus callosum. In the cuprizone model, Stidworthy et al. failed to find a role for Notch 1 in remyelination using a floxed Notch1 receptor and a tamoxifeninducible Cre driven by the myelin proteolipid protein (PLP) promoter (20). However, an alternative interpretation of their data could be that the target of both Jagged and F3/Contactin is an OPC at a developmental stage prior to expression of PLP. This would further be supported by the fact that developmental studies using a Cre driven by the 2 ', 3 ' - cyclic nucleotide 3 '-phosphodiesterase promoter that is expressed earlier than PLP have documented a role for canonical Notch1 signaling in oligodendrocyte differentiation (21). Clearly, additional animal studies that examine both the canonical and noncanonical pathways at earlier stages of OPC differentiation would be of interest.

Remyelinating areas of MS lesions (shadow plaques) are most commonly found at the edge of active lesions (1). Thus, microarray technology employing laser-capture tissue samples taken from the center and edge as well as adjacent normal-appearing white matter of such lesions may be a particularly informative experimental approach. Taken together, the data provided by Nakahara et al. (8) in this issue of JCI indicate that reexamining the role of Notch ligands and TIP30 in the adult CNS may be highly relevant to our understanding of the factors important in mediating myelin repair of MS lesions, which is critical for protection against axonal loss and recovery of function.

\section{Acknowledgments}

This work was supported by United States Public Health Service grants NS46620 and NS056074 (to G.R. John) and National Multiple Sclerosis Society Research grants RG3874 (to G.R. John) and RG3827 (to C.F. Brosnan).

Address correspondence to: Celia F. Brosnan, Departments of Pathology and Neuroscience, Albert Einstein College of Medicine, 1300 Morris Park Avenue, New York, New York 10461, USA. Phone: (718) 4302140; Fax: (718) 430-8541; E-mail: brosnan@aecom.yu.edu. Or to: Gareth R. John, Corinne Goldsmith Dickinson Center for Multiple Sclerosis, Department of Neurology, Annenberg 14-86, Box 11-37, Mount Sinai School of Medicine, 1 Gustave L. Levy Place, New York, New York 10029, USA. Phone: (212) 241-8142; Fax: (212) 3481310; E-mail: gareth.john@mssm.edu. 
1. Ludwin, S.K., and Raine, C.S. 2008. The neuropathology of MS. In Multiple sclerosis: a comprehensive text. C.S. Raine, H.F. McFarland, and R. Hohlfeld, editors. Saunders/Elsevier. New York, New York, USA. 151-177.

2. D’Souza, B., Miyamoto, A., and Weinmaster, G. 2008. The many facets of Notch ligands. Oncogene. 27:5148-5167.

3. Wang, S., et al. 1998. Notch receptor activation inhibits oligodendrocyte differentiation. Neuron. 21:63-75.

4. Popko, B. 2003. Notch Signaling: A rheostat regulating oligodendrocyte differentiation? Dev. Cell. 5:668-669.

5. Hu, Q.D., et al. 2003. F3/contactin acts as a functional ligand for Notch during oligodendrocyte maturation. Cell. 115:163-175.

6. Hu, Q.D., Ma, Q.-H., Gennarini, G., and Xiao, Z.C. 2006. Cross-talk between F3/Contactin and Notch at axoglial interface: A role in oligodendrocyte development. Dev. Neurosci. 28:25-33.

7. John, G.R., et al. 2002. Multiple sclerosis: re-expression of a developmental pathway that restricts oligodendrocyte maturation. Nat. Med. 8:1115-1121.

8. Nakahara, J., Kanekura, K., Nawa, M., Aiso, S., and Suzuki, N. 2009. Abnormal expression of TIP30 and arrested nucleocytoplasmic transport within oligodendrocyte precursor cells in multiple sclerosis. J. Clin. Invest. 119:169-181.

9. Padiath, Q.S., et al. 2006. Lamin B1 duplications cause autosomal dominant leukodystrophy. Nat. Genet. 38:1114-1123.

10. Schwankhaus, J.D., Katz, D.A., Eldridge, R., Schlesinger, S., and McFarland, H. 1994. Clinical and pathological features of an autosomal dominant, adult-onset leukodystrophy simulating chronic progressive multiple sclerosis. Arch. Neurol. 51:757-766.

11. King, F.W., and Shtivelman, E. 2004. Inhibition of nuclear import by the proapoptotic protein CC3. Mol. Cell. Biol. 24:7091-7101.

12. Lock, C., et al. 2002. Gene-microarray analysis of multiple sclerosis lesions yields new targets validated in autoimmune encephalomyelitis. Nat. Med. 8:500-508

13. El Omari, K., Bird, L.E., Nichols, C.E., Ren, J., and Stammers, D.K. 2005. Crystal structure of CC3 (TIP30): implications for its role as a tumor suppressor. J. Biol. Chem. 280:18229-18236.

14. Zhao, J., et al. 2008. Thirty-kilodalton Tat-interacting protein suppresses tumor metastasis by inhibition of osteopontin transcription in human hepatocellular carcinoma. Hepatology. 48:265-275.

15. Hur, E.M., et al. 2007. Osteopontin-induced relapse and progression of autoimmune brain disease through enhanced survival of activated T cells. Nat. Immunol. 8:74-83.

16. Ranscht, B. 1988. Sequence of contactin, a 130-kD glycoprotein concentrated in areas of interneuronal contact, defines a new member of the immunoglobulin supergene family in the nervous system. J. Cell Biol. 107:1561-1573.

17. Rios, J.C., et al. 2000. Contactin-associated protein (Caspr) and contactin form a complex that is targeted to the paranodal junctions during myelination. J. Neurosci. 20:8354-8364.

18. Wolswijk, G., and Balesar, R. 2003. Changes in the expression and localization of the paranodal protein Caspr on axons in chronic multiple sclerosis. Brain. 126:1638-1649.

19. Jurynczyk, M., Jurewicz, A., Raine, C.S., and Selmaj, K. 2008. Notch3 inhibition in myelin-reactive T cells down-regulates protein kinase $\mathrm{C}$ theta and attenuates experimental autoimmune encephalomyelitis. J. Immunol. 180:2634-2640.

20. Stidworthy, M.F., et al. 2004. Notch1 and Jagged1 are expressed after CNS demyelination, but are not a major rate-determining factor during remyelination. Brain. 127:1928-1941.

21. Genoud, S., et al. 2002. Notch1 control of oligodendrocyte differentiation in the spinal cord. J. Cell Biol. 158:709-718.

\title{
CD4+ $\mathrm{T}$ cells mediate cytotoxicity in neurodegenerative diseases
}

\author{
Stanley H. Appel
}

Department of Neurology, Methodist Neurological Institute, Houston, Texas, USA.

\begin{abstract}
Neuroinflammation, characterized by activated microglia and infiltrating $T$ cells, is a prominent pathological feature in neurodegenerative diseases. However, whether this inflammation contributes to neuronal injury or is a late consequence of neuronal injury is unclear. In this issue of the JCI, Brochard et al. report that $\mathrm{CD}^{+} \mathrm{T}$ cells are cytotoxic in a mouse model of Parkinson disease (PD) (see the related article beginning on page 182). Specifically, invading $T$ lymphocytes contributed to neuronal cell death via the Fas/FasL pathway. The results implicate the adaptive immune system in the pathogenesis of Parkinson neurodegeneration and provide a meaningful rationale for immune-based therapies for PD.
\end{abstract}

\section{Neuroinflammation in Parkinson disease}

Parkinson disease (PD) is a motor system disorder, which is characterized by tremor, rigidity, slowed movements, and impaired balance and coordination and results from the loss of dopamine-producing cells in the brain. The nigrostriatal pathway - a neural pathway connecting the substantia nigra

Conflict of interest: The author has declared that no conflict of interest exists.

Nonstandard abbreviations used: ALS, amyotrophic lateral sclerosis; MPTP, 1-methyl-4-phenyl-1,2,3,6tetrahydropyridine; PD, Parkinson disease; $\alpha$-Syn, $\alpha$-synuclein.

Citation for this article: J. Clin. Invest. 119:13-15 (2009). doi:10.1172/JCI38096. with the striatum - is one of four major dopamine signaling pathways in the brain and is prominently involved in controlling movement. In $\mathrm{PD}$, the nigrostriatal pathway as well as dopaminergic and nondopaminergic neurons are compromised, and this is accompanied by inflammatory changes in microglia (the innate immune cells of the central nervous system) and infiltration of $\mathrm{T}$ lymphocytes (cells of the adaptive immune system). It has long been thought that microglial and $\mathrm{T}$ cell infiltration are not primary events in the pathogenesis of neurodegeneration but are instead responses to neuronal injury. However, recent studies (discussed below) support an alternative point of view and provide compelling evi- dence that both activated microglia and $\mathrm{T}$ lymphocytes make a significant contribution to neurodegeneration, at the very least by amplifying and exacerbating an ongoing inflammatory process and by triggering extensive neuronal degeneration to develop from a small population of stressed dopaminergic neurons.

\section{The role of microglia in dopaminergic cytotoxicity}

Much attention has focused on microglia as one of the mediators of the inflammatory response leading to dopaminergic neuronal injury. Microglia are similar to macrophages and are capable of exhibiting either an M1 proinflammatory phenotype (following activation with LPS), characterized by the secretion of proinflammatory cytokines, NO, and superoxide, or an M2 antiinflammatory phenotype (following incubation with IL-4), characterized by the secretion of neurotrophic factors such as IGF-1 and IL-10 (1). In vitro, LPS-induced microglial activation triggers the release of proinflammatory factors, including $\mathrm{NO}, \mathrm{H}_{2} \mathrm{O}_{2}$, and superoxide, causing neurodegeneration of ventral midbrain dopaminergic neurons 